\title{
DISCONTINUITY THEOREM FOR FIRST ORDER PHASE TRANSITIONS. IMPLICATIONS FOR QCD*
}

\author{
A. Barducci, R. Casalbuoni and G. Pettini \\ Dipartimento di Fisica, Univ. di Firenze \\ I.N.F.N., Sezione di Firenze \\ R. Gatto \\ Département de Physique Théorique, Univ. de Genève \\ UGVA-DPT 1992/12-798 \\ December 1992 hep-ph/9212276
}

* Partially supported by the Swiss National Foundation 


\section{ABSTRACT}

A first order phase transition leading to deconfinement and chiral restoration is a likely possibility for QCD, at least in some region of the temperaturedensity plane. A signal for a unique transition is that the order parameters for such transitions (which can be understood in terms of symmetries only in limiting situations of very massive or massless quarks) are both discontinuous at the same critical temperature. We show that such a situation can be understood on a precise thermodynamical basis because of a general relation among discontinuities which holds for first order transitions. We derive the result by a generalization of the Clausius-Clapeyron equation and also through the effective action formalism. We illustrate the theorem in an elementary example. 


\section{Introduction and summary of conclusions}

There are two limiting situations in QCD where one expects the occurrence of phase transitions characterized by corresponding symmetry breaking. In the limit of infinite quark mass, the deconfinement transition can be related to the breaking of a discrete group $Z(3)$, which is a symmetry of the QCD lagrangian in that limit. The Polyakov loop acts as order parameter for the transition. In the opposite limit, of massless quarks, the chiral phase transition is related to restoration of chiral symmetry. The fermionic condensate acts as the relevant order parameter.

In the realistic case of QCD with quarks of finite mass, neither the discrete $Z(3)$ group, nor the global chiral symmetry are good symmetries of the lagrangian. One may however still consider to look at what happens to the two preceding order parameters, though they had been constructed for differing limiting situations.

A computer simulation, with 4 light quark flavors with dynamical mass $m / T \approx 0.1$, has shown an abrupt transition for the two order parameters at practically the same temperature [1], [2].

Another important issue is the order of the transition. Recent results, reviewed in ref. [3], point towards a first order phase transition in the case of two almost massless quarks and a massive one. It should also be mentioned that there appears to be a sort of critical value for the mass of the third quark, above which the transition becomes second order.

The problem of the transitions has also been studied in ref. [⿴囗十 assuming an effective lagrangian. The authors argue that the deconfined phase should be characterized by the vanishing of the gluonic condensate, $\left\langle 0\left|F_{\mu \nu} F^{\mu \nu}\right| 0\right\rangle \beta(g) /(2 g)=0$, and furthermore that the deconfinement transition should be first order. For this discussion it is convenient to introduce separate critical temperatures $T_{\chi}$ and $T_{d}$ for the chiral and the deconfinement transitions, as one would expect when ignoring couplings between different degrees of freedom. The authors of ref. [⿴囗 ㅇ] show that, if $T_{\chi}>T_{d}$, the first order deconfinement transition drives a discontinuity of the fermionic condensate. As a consequence the chiral transition appears of first order, whichever was its nature when ignoring other degrees of freedom, and at the same temperature of the deconfinement one. We also mention indications [5] that the topological susceptibility drives the transition of other quantities, such as the quark condensate or the Polyakov loop.

We shall prove here a general thermodynamical result. We shall show that a generalized version of the Clausius-Clapeyron equation relates discontinuities of the thermal averages of different observables at the critical point of a first order phase transition. In a more precise way, we consider a general hamiltonian, written in the form $H=\sum_{\alpha} \lambda_{\alpha} \hat{\mathcal{O}}_{\alpha}$, where $\lambda_{\alpha}$ are constants and $\hat{\mathcal{O}}_{\alpha}$ arbitrary operators. The critical point of the first order phase transition will depend, in general, on a subset of the parameters $\lambda_{\alpha}$, to be called $\left\{\lambda_{k}\right\}$. Then all the thermal averages of the operators $\hat{\mathcal{O}}_{k}$, related to the $\lambda_{k}$ 's, are expected to become discontinuous at the critical point, both in their dependence from temperature and from density.

The consequence for QCD is obvious, assuming that there is a first order transition and that the critical point depends on parameters, such as quark masses, the QCD coupling constant, etc. Then the quark condensate, the gluon condensate, etc., are expected to

exhibit simultaneous discontinuities at such a critical point. Of course, this is not a 
proof that such a scenario necessarily occurs in QCD, but it clearly shows that such an apparently curious thing as a unique phase transition, perceived through order parameters related to different, even incompatible, limiting situations of the theory, has a simple and precise thermodynamical basis.

For the derivation we first present a generalization of the Clausius-Clapeyron equation (section 2). In section 3 we deal with the discontinuities. We give a derivation based on the functional effective action formalism in section 4. A most elementary example, developed in section 5 , illustrates the simplicity of the result.

\section{Generalization of the Clausius-Clapeyron relation}

To derive the relation we are interested in, let us start by recalling some thermodynamical relations. Consider the partition function in the Grand Canonical Ensemble

$$
Z=\operatorname{Tr} e^{-\beta(\hat{H}-\mu \hat{N})}
$$

and a Hamiltonian of the form

$$
\hat{H}=\hat{H}_{0}+\sum_{\alpha=1}^{N} \lambda_{\alpha} \hat{\mathcal{O}}_{\alpha}
$$

where $\lambda_{\alpha}$ are real parameters conjugate to the hermitean operators $\hat{\mathcal{O}}_{\alpha}$. The expectation value of the operator $\hat{\mathcal{O}}_{\alpha}$ is

$$
\begin{aligned}
\left\langle\hat{\mathcal{O}}_{\alpha}\right\rangle & =-\frac{\partial(T \ln Z)}{\partial \lambda_{\alpha}} \\
& =\frac{1}{Z} \operatorname{Tr}\left[\hat{\mathcal{O}}_{\alpha} e^{-\beta(\hat{H}-\mu \hat{N})}\right]
\end{aligned}
$$

In the same way, by varying with respect to the chemical potential $\mu$, one has the mean particle number (or it could be any conserved charge, and we are not necessarily considering a fermionic theory)

$$
\langle\hat{N}\rangle=\frac{\partial(T \ln Z)}{\partial \mu}
$$

We also recall that the entropy $S$ is

$$
S=\frac{\partial(T \ln Z)}{\partial T}
$$

Now, let us assume that the theory describes a system which can exist in two phases, each described by an equation of state $p_{i}=p_{i}(T, \mu) \quad(i=1,2)$, where $p_{i}$ is the pressure in the particular phase; and that there exists a set of points $\left(\mu_{c}, T_{c}\right)$ in the plane of chemical potential and temperature, by crossing which the system undergoes first order

phase transitions. In any of these points the equations of state of the two phases coincide

$$
p_{1}\left(T_{c}\left(\left\{\lambda_{k}\right\}\right), \mu_{c}\left(\left\{\lambda_{k}\right\}\right),\left\{\lambda_{k}\right\}\right)=p_{2}\left(T_{c}\left(\left\{\lambda_{k}\right\}\right), \mu_{c}\left(\left\{\lambda_{k}\right\}\right),\left\{\lambda_{k}\right\}\right)
$$


$\left(\left\{\lambda_{k}\right\}\right.$ indicates the subset of parameters which affect the critical behaviour).

If the pressure depends smoothly on $\left\{\lambda_{k}\right\}$, we can require the equality of the total derivatives of the two members in eq. (2.6) with respect to $\lambda_{k}$

$$
\left.\frac{d p_{1}}{d \lambda_{k}}\right|_{c}=\left.\frac{d p_{2}}{d \lambda_{k}}\right|_{c}
$$

obtaining

$$
\left.\frac{\partial p_{1}}{\partial \lambda_{k}}\right|_{c}+\left.\frac{\partial p_{1}}{\partial T}\right|_{c} \frac{d T_{c}}{d \lambda_{k}}+\left.\frac{\partial p_{1}}{\partial \mu}\right|_{c} \frac{d \mu_{c}}{d \lambda_{k}}=\left.\frac{\partial p_{2}}{\partial \lambda_{k}}\right|_{c}+\left.\frac{\partial p_{2}}{\partial T}\right|_{c} \frac{d T_{c}}{d \lambda_{k}}+\left.\frac{\partial p_{2}}{\partial \mu}\right|_{c} \frac{d \mu_{c}}{d \lambda_{k}}
$$

Here the subscript $\left.\right|_{c}$ means that the quantities are evaluated at $\left(\mu_{c}, T_{c}\right)$. We recall that according to the Ehrenfest classification, in a first order phase transition the pressure (or any another state function) is continuous whereas its first (partial) derivatives are discontinuous. Also, in the thermodynamic limit

$$
p \mathrm{~V}=T \ln Z
$$

(here $\mathrm{V}$ is the three-dimensional volume and the pressure has been taken vanishing at $T=\mu=0$ ) Thus eq. (2.8) can be written in the form

$$
\operatorname{disc}\left\langle\hat{o}_{k}\right\rangle=\frac{d T_{c}}{d \lambda_{k}} \operatorname{disc} s+\frac{d \mu_{c}}{d \lambda_{k}} \operatorname{disc} n
$$

where

$$
\left\langle\hat{o}_{k}\right\rangle=\frac{\left\langle\hat{\mathcal{O}}_{k}\right\rangle}{\mathrm{V}} \quad s=\frac{S}{\mathrm{~V}} \quad n=\frac{\langle\hat{N}\rangle}{\mathrm{V}}
$$

Let us now imagine two lines of first order critical points, one at $\lambda_{k}$ and another at $\lambda_{k}+d \lambda_{k}$. If we consider eq. (2.10) at $\left(\mu_{c}, T_{c}\right)$ on the line at $\lambda_{k}$, we can unambiguously evaluate the discontinuities of the densities $\left\langle\hat{o}_{k}\right\rangle, s$, and $n$, whereas we have to specify on which point $\left(\mu_{c}^{\prime}, T_{c}^{\prime}\right)$ of the line at $\lambda_{k}+d \lambda_{k}$ we end in order to calculate the two derivatives $d T_{c} / d \lambda_{k}$ and $d \mu_{c} / d \lambda_{k}$. In other words, although these derivatives are bound to satisfy eq. (2.10), we have the freedom to choose the direction along which to evaluate them. In particular we can consider the variation of $T_{c}$ ( of $\mu_{c}$ ) with respect to $\lambda_{k}$ at fixed $\mu$ (at fixed $T$ ), and in this way we can express disc $\left\langle\hat{o}_{k}\right\rangle$ only in terms of of disc $s$ (of disc $n$ ), eliminating the other discontinuity. Thus, under these conditions, we have the further relation

$$
\left.\frac{\partial T_{c}}{\partial \lambda_{k}}\right|_{\mu} \operatorname{disc} s=\left.\frac{\partial \mu_{c}}{\partial \lambda_{k}}\right|_{T} \operatorname{disc} n
$$

The previous result can be also expressed by the fact that, in presence of a critical line, $T_{c}$ and $\mu_{c}$ are not independent variables and that if we fix for instance the chemical potential $d T_{c} / d \lambda_{k}=\partial T_{c} / \partial \lambda_{k}$

Let us now consider the thermodynamic relation

$$
\begin{aligned}
\varepsilon & =-p+T \frac{\partial p}{\partial T}+\mu \frac{\partial p}{\partial \mu} \\
& =-p+T s+\mu n
\end{aligned}
$$

which allows to determine the latent heat for a first order phase transition

$$
\operatorname{disc} \varepsilon=T_{c} \operatorname{disc} s+\mu_{c} \operatorname{disc} n
$$


By using eqs. (2.10) and (2.12), disc $\varepsilon$ can be entirely expressed in terms of disc $\left\langle\hat{o}_{k}\right\rangle$

$$
\operatorname{disc} \varepsilon=\left(\frac{1}{\partial\left(\ln T_{c}\right) /\left.\partial \lambda_{k}\right|_{\mu}}+\frac{1}{\partial\left(\ln \mu_{c}\right) /\left.\partial \lambda_{k}\right|_{T}}\right) \operatorname{disc}\left\langle\hat{o}_{k}\right\rangle
$$

The relation (2.15) is of the type of the Clausius-Clapeyron equation for a liquid-vapor first order transition,

$$
\frac{d T_{c}}{d p}=\frac{\operatorname{disc} v}{\operatorname{disc} s}
$$

( $v$ is the specific volume) as shown in a recent paper by H.Leutwyler [6] for zero chemical potential.

However the relation (2.15) is quite more general; it concerns any operator and it allows for a chemical potential. We must remember that to get (2.15) in this precise form, eq. (2.12) is necessary, which requires the existence of lines of first order critical points at varying parameters $\{\lambda\}$, rather than an isolated critical point.

\section{Relations among discontinuities}

Eqs. (2.10), (2.12) (and (2.15)) hold for the thermal average of any operator whose conjugate parameter influences the critical point. If $\hat{\mathcal{O}}_{i}$ and $\hat{\mathcal{O}}_{j}$ are two of such operators, eliminating alternatively disc $s$ and disc $n$ from eqs. (2.10) and (2.12), written by varying with respect to the parameters $\lambda_{i}$ and $\lambda_{j}$, we also get

$$
\begin{aligned}
& \operatorname{disc}\left\langle\hat{o}_{i}\right\rangle=\frac{\partial T_{c} /\left.\partial \lambda_{i}\right|_{\mu}}{\partial T_{c} /\left.\partial \lambda_{j}\right|_{\mu}} \operatorname{disc}\left\langle\hat{o}_{j}\right\rangle \\
& \operatorname{disc}\left\langle\hat{o}_{i}\right\rangle=\frac{\partial \mu_{c} /\left.\partial \lambda_{i}\right|_{T}}{\partial \mu_{c} /\left.\partial \lambda_{j}\right|_{T}} \operatorname{disc}\left\langle\hat{o}_{j}\right\rangle
\end{aligned}
$$

Namely, all the operators conjugate to parameters which affect the critical point are expected to be discontinuous at the phase transition point. The ratio of their discontinuities is simply the ratio of the variation of the critical temperature or chemical potential with respect to the correspondent conjugate parameters. From the two previous equations one also gets

$$
\frac{\partial T_{c} /\left.\partial \lambda_{i}\right|_{\mu}}{\partial T_{c} /\left.\partial \lambda_{j}\right|_{\mu}}=\frac{\partial \mu_{c} /\left.\partial \lambda_{i}\right|_{T}}{\partial \mu_{c} /\left.\partial \lambda_{j}\right|_{T}}
$$

Of course we could have employed other variables instead of the pressure and followed the procedure starting from eq. (2.6) obtaining relations similar to eq. (2.15). For instance, by equating the chemical potentials $\mu_{i}(p, T)$ in the two phases for the liquidvapor transition, varying with respect to $p\left(T_{c}\right.$ is a function of $\left.p\right)$, one obtains eq. (2.16). The reason why we have chosen the pressure will be clearer in the following.

In this context our aim is to stress the essential ingredients. If in some system a first order phase transition occurs, using the fact that the state functions are continuous, but not their partial derivatives, one can easily relate the various discontinuities appearing in the description, obtaining alternative expressions for quantities such as the latent heat. 
In particular these relations can be useful, as already remarked by Leutwyler [6], if one can easier compute the discontinuity of some operator rather than the discontinuities of the derivatives of the pressure.

The previous analysis can readily be extended to cases where there is a set of chemical potentials $\mu_{i}$ coupled to charge operators $\hat{N}_{i}$.

\section{The effective action derivation}

Let us consider a field theory at finite temperature $T$ and chemical potential $\mu$, described by an euclidean effective action $\Gamma$. The effective action is a functional of a set of operators $\{\hat{A}\}$, the dynamical variables of the theory,

$$
\Gamma=\Gamma[\{\hat{\mathrm{A}}\} ;\{\alpha\} ; T, \mu]
$$

In the previous equation we have also explicitly written the dependence of $\Gamma$ on a set of parameters $\{\alpha\}$, which are external sources conjugate to the operators $\{\hat{A}\}$. Suppose that by varying the temperature $T$ and/or the chemical potential $\mu$, the effective action can describe different phases of the theory, and that there exist lines of first order critical points $\left(T_{c}, \mu_{c}\right)$ in the $(T, \mu)$ plane. Then in any of these points $\left(T_{c}, \mu_{c}\right), \Gamma$ has two degenerate absolute minima $\{\hat{A}\}_{1},\{\hat{A}\}_{2}$, where we can write the condition

$$
\Gamma\left[\{\hat{A}\}_{1} ;\{\alpha\} ; T_{c}(\{\alpha\}), \mu_{c}(\{\alpha\})\right]=\Gamma\left[\{\hat{A}\}_{2} ;\{\alpha\} ; T_{c}(\{\alpha\}), \mu_{c}(\{\alpha\})\right]
$$

Then we equate the total derivatives with respect to any parameter $\alpha_{k}$ which affects the critical behaviour, getting, apart from terms continuous at the transition,

$$
\left.\left[\frac{\partial \Gamma}{\partial \alpha_{k}}+\frac{\partial \Gamma}{\partial T} \frac{d T_{c}}{d \alpha_{k}}+\frac{\partial \Gamma}{\partial \mu} \frac{d \mu_{c}}{d \alpha_{k}}+\frac{\partial \Gamma}{\partial\{\hat{A}\}} \frac{d\{\hat{A}\}}{d \alpha_{k}}\right]\right|_{c} ^{\{A\}_{1}}=\left.(\text { same })\right|_{c} ^{\{A\}_{2}}
$$

The derivatives of $\Gamma$ with respect to $\{\hat{A}\}$ vanish on both sides of the previous expression, since they are evaluated at the two minima of the effective action. Actually the effective action evaluated at these points is nothing but $-\ln Z$ and therefore $-p V / T$ (since it is defined for infinite volume) for the two coexisting phases. Thus at the physical point it looses its dependence on the dynamical variables, whereas it remains a function of the sources $\{\alpha\}$. Furthermore, in the case of constant fields, we can employ the euclidean effective potential

$$
\mathcal{V}=\frac{\Gamma}{\beta V}=-p
$$

which directly gives the pressure.

It follows that eq. (4.3), written for the effective potential, is equivalent to eq. (2.8). 


\section{A simple example}

Let us now consider a simple example. It is almost trivial, as it involves a single field. However it is useful to understand how the theorem works.

Consider an effective potential containing powers up to the sixth order of a scalar field $\phi$, which plays the role of an order parameter [7]:

$$
\mathcal{V}=a \phi^{2}-b \phi^{4}+c \phi^{6}+h \phi
$$

where we assume $c>0$ and $h$ small. We will show how the equations $(2.10),(2.15)$ are satisfied in this particular case, by taking $h$ as the parameter $\lambda$ to be varied. Of course we could use any of the other parameters appearing in eq. (5.1) but we will limit ourselves to this case. At the end of the section we will discuss also eq. (3.1) and then we will also use the other parameters appearing in eq. (5.1) as variable parameters $\lambda_{i}$. We start by discussing the model at $h=0$. The potential has a critical line of second-order transitions for $b<0$ and $a=0$. At $a=b=0$ the model has a tricritical point [8], whereas for $b>0$ there is a line of first-order transitions given by the equation

$$
a=\frac{b^{2}}{4 c}
$$

The tricritical point is where the two lines of first- and second-order transitions merge together. At any of the first-order transition points one has two phases characterized by the following values of $\phi$ (interpreted as the vacuum expectation values, v.e.v.)

$$
\langle\phi\rangle_{1}=0, \quad\langle\phi\rangle_{2}=\sqrt{\frac{b}{2 c}}
$$

We also define the discontinuity as

$$
\operatorname{disc}\langle\phi\rangle=\langle\phi\rangle_{2}-\langle\phi\rangle_{1}=\sqrt{\frac{b}{2 c}}
$$

For the following discussion we will assume also a linear dependence of the parameter $a$ on the temperature and on the chemical potential around the first-order critical line. We could assume an analogous dependence for any of the parameters, but to keep the matter simple we will stay with $a$. We put

$$
a=\alpha T+\gamma \mu-a_{0}
$$

obtaining for the critical line

$$
\alpha T_{c}+\gamma \mu_{c}=a_{0}+\frac{b^{2}}{4 c}
$$

When we insert the small perturbation $h \phi$ we need (at the lowest order in $h$ ) only to know how the critical quantities are changed

$$
\alpha T_{c}+\gamma \mu_{c}=a_{0}+\frac{b^{2}}{4 c}-\sqrt{\frac{2 c}{b}} h
$$

By noticing that in this case the pressure is given by $p=-\mathcal{V}$, we get for the specific entropy and for the density

$$
s=-\alpha\left\langle\phi^{2}\right\rangle, \quad n=-\gamma\left\langle\phi^{2}\right\rangle
$$


By taking $\lambda=h$ we can verify eq. (2.10)

$$
\begin{aligned}
& \frac{d T_{c}}{d h} \operatorname{disc} s+\frac{d \mu_{c}}{d h} \operatorname{disc} n= \\
= & \left(-\frac{1}{\alpha} \sqrt{\frac{2 c}{b}}-\frac{\gamma}{\alpha} \frac{d \mu_{c}}{d h}\right)\left(-\alpha \operatorname{disc}\left\langle\phi^{2}\right\rangle\right)+\frac{d \mu_{c}}{d h}\left(-\gamma \operatorname{disc}\left\langle\phi^{2}\right\rangle\right)= \\
= & \sqrt{\frac{2 c}{b}} \frac{b}{2 c}=\operatorname{disc}\langle\phi\rangle
\end{aligned}
$$

From eq. (5.8) and (2.14) we can derive the discontinuity for the energy density

$$
\operatorname{disc} \epsilon=\left(-\alpha T_{c}-\gamma \mu_{c}\right) \operatorname{disc}\left\langle\phi^{2}\right\rangle
$$

Then, by differentiating (5.7)

$$
\left.\frac{\partial T_{c}}{\partial h}\right|_{\mu}=-\frac{1}{\alpha \operatorname{disc}\langle\phi\rangle},\left.\quad \frac{\partial \mu_{c}}{\partial h}\right|_{T}=-\frac{1}{\gamma \operatorname{disc}\langle\phi\rangle}
$$

it is simple matter to verify eq. (2.15). Finally we verify eq. (3.1) by taking $h$ and $c$ as $\lambda$ parameters. Using

$$
\operatorname{disc}\left\langle\phi^{6}\right\rangle=\left(\frac{b}{2 c}\right)^{3}
$$

we get (in the limit $h \rightarrow 0$ )

$$
\begin{aligned}
\operatorname{disc}\left\langle\phi^{6}\right\rangle & =\frac{\partial T_{c} /\left.\partial c\right|_{\mu}}{\partial T_{c} /\left.\partial h\right|_{\mu}} \operatorname{disc}\langle\phi\rangle= \\
& =\left(-\frac{1}{\alpha} \frac{b^{2}}{4 c^{2}}\right)\left(-\frac{1}{\alpha} \sqrt{\frac{2 c}{b}}\right)^{-1} \sqrt{\frac{b}{2 c}}=\left(\frac{b}{2 c}\right)^{3}
\end{aligned}
$$

All the other relations can be proved in analogous way. 


\section{References}

[1] F.Karsch, J.Kogut, D.K.Sinclair and H.W.Wyld, Phys. Letters 188B (1987) 353

[2] For a review see: H.Satz, Proceedings of the Aachen Workshop on LHC, Aachen, 4-9 October 1990, CERN 90-10, ECFA 90-133, Eds. G.Jarlskog and D.Rein, Vol. 1, p. 188

[3] B.Petersson, Proceedings of the Eight International Conference on Ultrarelativistic Nucleus-Nucleus Collisions, Quark Matter 1990, Menton, France, May 7-11, 1990, Eds. J.P.Blaizot, C.Garschel, B.Pire and A.Romana, Nucl. Physics A525 (1991) $237 \mathrm{c}$

[4] B.Campbell, J.Ellis and K.A.Olive, Nucl. Physics B345 (1990) 57

[5] H.Gausterer, J.Potvin and S.Salenievici, Phys. Review D41 (1990) 3829

[6] H.Leutwyler, Phys. Letters 284B (1992) 106

[7] For a discussion of this model see: K.Huang, "Statistical Mechanics", $2^{\text {nd }}$ edition, edited by J.Wiley and Sons, p. 428 (1987)

[8] We follow the terminology suggested by R.B.Griffiths, Phys. Rev. Letters 24 (1970) 715 